\title{
Sentido del humor, afectos y personalidad. Estudio en estudiantes universitarios
}

\section{Sense of Humor, Affect, and Personality. A Study of University Students Sentido de humor, afetos e personalidade. Estudo em estudantes universitários}

\author{
Carlos Salavera*, Pablo Usán, Laurane Jarie, Orosia Lucha \\ Universidad de Zaragoza
}

Doi: http://dx.doi.org/10.12804/revistas.urosario.edu.co/apl/a.4511

\section{Resumen}

En el presente estudio, se evaluó a 652 estudiantes universitarios para analizar la relación entre el uso del humor, los afectos y su personalidad. Los resultados muestran cómo los hombres hacen un mayor uso del humor, tanto positivo como negativo, y presentan mayores niveles de impulsividad, actividad, sociabilidad y agresividad, no encontrándose diferencias en el manejo de los afectos. Las personas que muestran un mayor uso del humor positivo (afiliativo y automejora) también utilizan más los afectos positivos y muestran mayores niveles de actividad, sociabilidad e impulsividad. Por último, los resultados indican cómo tan solo 293 participantes $(44,93 \%)$ hacen un buen uso de los afectos, mayor uso de los estilos de humor positivo y presentan bajos niveles de ansiedad; 171 personas (26,22\%) muestran bajos niveles en los afectos y alto uso del humor afiliativo y agresivo como método de sociabilidad; y un grupo de 188 personas $(28,83 \%)$ tiene altos afectos negativos, bajo uso de los estilos de humor positivos y alta ansiedad y agresividad. Los datos proporcionan evidencia de la relación entre el sentido del humor, los afectos y la personalidad.

Palabras clave: sentido del humor, afectos positivo y negativo, personalidad.

\section{fbstract}

In the present study, 652 college students were evaluated to analyze the relationship between the use of humor, affect, and personality. The results showed that males made greater use of both positive and negative humor and had higher levels of impulsivity, activity, sociability, and aggressiveness. No differences between the sexes were found in the management of affect. People who showed greater use of positive humor (affiliative and self-enhancing), also showed more positive affect and higher levels of activity, sociability, and impulsivity. Finally, 293 participants (44.93\%) showed positive affect, increased positive mood, and low levels of anxiety; 171 people (26.22\%) exhibited lower affect and high affiliative and aggressive use of humor as a method of sociability, and 188 people $(28.83 \%)$ had highly

* Correspondencia. Correo electrónico: salavera@unizar.es

Cómo citar este artículo: Salavera, B. C., Usán, P., Jarie, L., Lucha, O. (2018). Sentido del humor, afectos y personalidad. Estudio en estudiantes universitarios. Avances en Psicología Latinoamericana, 36(1), 83-91. doi: http://dx.doi.org/10.12804/ revistas.urosario.edu.co/ap1/a.4511 
negative affect, little exhibition of positive mood, and styles characterized by high anxiety and aggressiveness. The data provide evidence of the relationship between sense of humor, affect, and personality.

Keywords: Sense of humor, positive and negative affect, personality.

\section{Resumo}

No presente estudo, avaliaram-se 652 estudantes universitários para analisar a relação entre o uso do humor, os afetos e sua personalidade. Os resultados mostram como os homens fazem um maior uso do humor, tanto positivo quanto negativo e apresentam maiores níveis de impulsividade, atividade, sociabilidade e agressividade, não se encontrando diferenças no manejo dos afetos. As pessoas que mostram um maior uso do humor positivo (afiliativo e auto-reforço), também utilizam mais os afetos positivos e mostram maiores níveis de atividade, sociabilidade e impulsividade. Por último, os resultados indicam como tão só 293 participantes $(44,93 \%)$ fazem um bom uso dos afetos, maior uso dos estilos de humor positivo e apresentam baixos níveis de ansiedade; 171 pessoas $(26,22 \%)$ mostram baixos níveis nos afetos e alto uso do humor afiliativo e agressivo, como método de sociabilidade; encontrando-se um grupo de 188 pessoas $(28,83 \%)$ com altos afetos negativos, sob uso dos estilos de humor positivos e alta ansiedade e agressividade. Os dados proporcionaram evidência da relação entre o sentido de humor, os afetos e a personalidade.

Palavras-chave: sentido de humor, afetos positivo e negativo, personalidade.

El sentido del humor ha cobrado especial importancia en los últimos años debido a su relación con la salud física y psicológica de la persona (Capps, 2006; Kuiper, 2014), encontrándose relación con la satisfacción con la vida, reducción del dolor, mejora de la condición física y mejores niveles de salud en general (Cann \& Collette, 2014; Panish,
2002). La idea de que la risa y el humor fomentan la salud no es nueva, pero en las últimas décadas algunos casos célebres de "curaciones", mediante el consumo y/o producción de material cómico (Cousins, 1979), la proliferación de diversas terapias e intervenciones clínicas relacionadas con el humor y el estudio científico de estos fenómenos ha generado un considerable interés mediático y público en torno al tema. En cuanto a pruebas empíricas, actualmente puede decirse que existen algunos indicios del valor terapéutico que se atribuye al humor, pero aún es demasiado pronto para afirmar que la risa es "la mejor medicina" (Baquero \& Jáuregui, 2006), aunque hay que señalar que el humor está implicado en algunas de las tareas psicológicas más complejas e importantes, siendo responsable de distintos beneficios sociales, cognitivos y del comportamiento, tanto en tareas individuales como en aspectos interpersonales (Cassaretto \& Martínez, 2009; Gignac, Karatamoglou, Wee \& Palacios, 2014; Kozbetz \& Nishioka, 2010). Autores como Fernández-Abascal (2009) consideran al humor como una forma específica del estado afectivo y emocional, lo que denomina un "tono emocional base". Divide el humor en una serie de dimensiones: dimensión cognitiva, comportamental, afectiva y social. Por otra parte, autores como Martin (2001) hablan de diferentes estilos de humor. Dos que son, a priori, potencialmente beneficiosos para la persona que los posee: afiliativo (uso benigno del humor para facilitar las relaciones interpersonales) y automejora (empleo de una visión humorística pero realista de la vida); y dos que son considerados potencialmente perjudiciales: agresivo (utilizado para sobresalir a expensas de otros) y autoagresivo (uso de un humor cínico divirtiendo a los demás a expensas de la propia persona).

De otra parte, el afecto se ha definido en torno a dos disposiciones ortogonales: una bipolaridad afecto positivo/afecto negativo, sustentada en una base hereditaria (Watson \& Tellegen, 1985). 
Mientras el afecto positivo ocupa la dimensión de emociones placenteras: motivación, energía, deseo de afiliación, logro o éxito; el afecto negativo representa el malestar y la emoción displacentera: miedos, inhibiciones, inseguridad, frustración y fracaso (Longo, 2015; Watson, Clark \& Tellegen, 1998). Así, una persona con alto afecto positivo acostumbra a experimentar sentimientos de satisfacción, entusiasmo, energía, amistad, unión, afirmación y confianza. Relacionado con la extroversión, el optimismo y la resiliencia. Y una persona con alto afecto negativo suele experimentar desinterés, aburrimiento, tristeza, culpa, vergüenza y envidia. Tienen una sensibilidad temperamental ante estímulos negativos: labilidad vegetativa, estresores y ambientes desfavorables (Clark \& Watson, 1991; Crawford \& Henry, 2004).

En cuanto a la personalidad, se puede definir como el conjunto de características y diferencias individuales que diferencian a una persona de otra. A este respecto, se ha estudiado mucho en los últimos años, con grandes aportaciones a este constructo, como el modelo de los cinco factores (McRae \& Costa, 1987) o el modelo de los cinco alternativos: neuroticismo-anisedad, agresión-hostilidad, actividad, sociabilidad y búsqueda de sensaciones impulsiva no socializada (Zuckerman, 2005; Zuckerman, Kuhlman, Joireman, Teta \& Kraft, 1993), con una base consistente en términos biológico-evolutivos.

Algunos autores han sugerido que tanto los afectos como el humor cumplen un papel importante en las relaciones interpersonales, que pueden estar relacionadas con la personalidad del individuo (Aillaud \& Piolat, 2013; Kuiper, Grimshaw, Leite \& Kirsh, 2004; Moran, Rain, Page-Gould \& Mar, 2014; Yip \& Martin, 2006).

El objetivo de esta investigación ha estado centrado en el análisis de la relación de los diferentes estilos de humor (afiliativo, de automejora, agresivo y autoagresivo), con los afectos (positivo y negativo) y la personalidad del individuo.

\section{Método}

\section{Participantes}

La muestra estuvo formada por 652 estudiantes de una Universidad pública española: 111 hombres $(17,02 \%)$ y 541 mujeres $(82,98 \%)$, que participaron en el estudio de manera voluntaria. Los participantes en la investigación cursaban estudios de Terapia Ocupacional, Fisioterapia, Enfermería, Trabajo Social, Magisterio de Educación Primaria y Magisterio de Educación Infantil. Todos ellos firmaron el consentimiento informado; además, se observaron las consideraciones éticas de la Declaración de Helsinki y se cumplieron los criterios éticos de investigación con seres humanos (consentimiento informado, derecho de información, protección de datos personales y garantías de confidencialidad, no discriminación, gratuidad y posibilidad de abandonar el estudio en cualquiera de sus fases). La edad de los participantes fue de 18 a 30 años, con una media de 20,73 años (d.s. $=0,811)$.

\section{Instrumentos}

Cuestionario de estilos de humor (HSQ) (Martin, Publio-Doris, Larsen, Gray \& Weir, 2003)

Este cuestionario de estilos de humor tiene formato de autoinforme con 32 ítems, de escala Likert de 5 puntos, que señala cuatro estilos de humor (afiliación: uso benigno del humor para facilitar las relaciones interpersonales; automejora: empleo de una visión humorística pero realista de la vida; agresivo: utilizado para sobresalir a expensas de otros y autodestructivo: uso de un humor cínico divirtiendo a los demás a expensas de la propia persona). En la presente investigación, la fiabilidad de las escalas fue: Afiliación (alfa $=0,80$ ); automejora (alfa $=0,82)$; agresivo $($ alfa $=0,78)$; y autodestructivo $($ alfa $=0,79)$. 
Cuestionario PANAS (Watson, Clark \& Tellegen, 1988)

La escala de Afecto Positivo y Negativo PANAS incluye 20 ítems, 10 de los cuales se refieren a la subescala de afecto positivo (AP) y 10 a subescala de afecto negativo (AN), en una escala tipo likert. Todos ellos están referidos al momento de contestar la escala, y se valoran de 0 (ausencia de la emoción) hasta 5 (presencia frecuente de la emoción). Esta escala presentó un alfa de 0,90 para el afecto positivo y de 0,87 para el afecto negativo.

ZKPQ-50-CC (Aluja, Rossier, García, Angleitner, Kuhlman \& Zuckerman, 2006)

El cuestionario ZKPQ-50-CC es una escala que evalúa mediante autoinforme la personalidad del sujeto. Compuesto por 50 ítems, en formato dicotómico (verdadero-falso). Este instrumento evalúa las diferentes dimensiones de la personalidad siguiendo el modelo de los Cinco Alternativos de Zuckerman et al. (1993): neuroticismo-ansiedad, impulsividad-búsqueda de sensaciones, actividad, sociabilidad y agresión-hostilidad. En el estudio se obtuvo una alta consistencia interna $(\mathrm{alfa}=0,78)$.

\section{Procedimiento}

Los participantes tenían una hora para completar los cuestionarios que se acaban de describir y el consentimiento informado. En todos los casos se les recordaba que la información recogida era anónima y confidencial. Los datos recogidos en el presente estudio se obtuvieron entre los meses de octubre a diciembre de 2014.

Para el análisis estadístico de los datos se ha empleado el programa estadístico SPSS 22.0. Comprobada la normalidad de la muestra e igualdad de varianzas se optó por técnicas paramétricas. Se realizó un análisis descriptivo para cada una de las variables. En todos los casos se trabajó con el menor nivel de significación posible y se han considerado significativas aquellas diferencias con un valor de $p<0,05$. Los contrastes se plantearon de forma bilateral. Se estableció un conglomerado de medias para obtener clúster que permitiesen clasificar a los participantes en distintos grupos en función de sus afectos, sentido del humor y personalidad. Finalmente, el establecimiento de ecuaciones predictoras de afectos positivos y negativos en función de la personalidad se realizó mediante la técnica de regresión logística, siguiendo el procedimiento de regresión por pasos hacia delante basado en el estadístico de Wald.

\section{Resultados}

Se analizaron las diferencias respecto al género en las tres variables medidas: sentido del humor, afectos y personalidad (tabla 1). Se encontraron diferencias significativas en todos los cuatro estilos del humor, así como en personalidad (excepto en el apartado de neuroticismo-ansiedad). Sin embargo, el género no mostró ninguna influencia en la expresión de los afectos medidos con la escala PANAS.

En los distintos factores del humor, las puntuaciones indican un mejor manejo de este en sus cuatro factores por parte de los hombres. Mientras, los resultados de la prueba de personalidad muestran cómo los hombres presentaron índices más altos en los cinco factores (ansiedad, impulsividad, actividad, sociabilidad y agresividad). Sin embargo, aun cuando los hombres mostraban niveles más altos en afectos negativos, estas diferencias no resultaron significativas. En la escala PANAS las personas con puntuaciones más altas de 38 en positivo y por debajo de 16 en negativo se caracterizan por una balanza de afectos extremadamente positiva, mientras que personas con puntuaciones por debajo de 25 en positivo y por encima de 30 en negativo muestran una balanza extremadamente negativa (Páez, 2008). En este punto, un $0,92 \%$ de los hombres y un 3,51\% de las mujeres, tenían afectos extremadamente positivos; por otra parte, un $4,50 \%$ de los hombres y 
un $7,20 \%$ de las mujeres se sitúan en afectos extremadamente negativos, cuestión muy relevante al tratarse de una muestra no clínica.

La tabla 2 presenta las correlaciones entre la escala de afectos (PANAS), los estilos del Humor (HSQ) y la personalidad (ZKPQ-50-CC).

En cuanto a los estilos de humor, todos ellos muestran relación con aspectos de personalidad y humor, se destaca que en el caso de neuroticismo-ansiedad esta relación es negativa y en el caso de la actividad no se encontró relación con los tipos de humor, a excepción del humor de automejora.
En el caso de los afectos y el sentido del humor, se encontraron correlaciones de los afectos positivo y negativo con los estilos del humor afiliativo y de automejora, siendo inversa en el caso del afecto negativo. No se encontró relación entre los afectos y el humor agresivo y autodestructivo (a excepción de este con el afecto negativo).

Por último, se realizó un análisis de clúster (tabla 3) para intentar clasificar a los participantes en la investigación en función de las tres variables: afectos, sentido del humor y personalidad. De esta manera, se obtuvieron tres grupos: (i) formado

Tabla 1

Resultados por género en las escalas HSQ, PANAS Y ZKPQ-50-CC

\begin{tabular}{|c|c|c|c|c|c|c|c|}
\hline & & \multicolumn{2}{|c|}{ Hombres } & \multicolumn{2}{|c|}{ Mujeres } & \multirow[b]{2}{*}{$\mathrm{t}$} & \multirow[b]{2}{*}{ Signif. } \\
\hline & & $\mathrm{x}$ & d.s & $\mathrm{x}$ & d.s & & \\
\hline \multirow{4}{*}{ HSQ } & Humor afiliativo & 33,62 & 3,92 & 31,44 & 4,47 & 4,786 & 0,000 \\
\hline & Humor automejora & 28,29 & 5,00 & 25,99 & 5,03 & 4,397 & 0,000 \\
\hline & Humor agresivo & 21,01 & 3,58 & 18,56 & 3,87 & 6,139 & 0,000 \\
\hline & Humor autodestructivo & 20,08 & 4,74 & 16,36 & 4,25 & 8,212 & 0,000 \\
\hline \multirow{2}{*}{ PANAS } & Afectos positivos & 32,40 & 6,82 & 32,40 & 6,22 &, 008 & 0,994 \\
\hline & Afectos negativos & 21,21 & 8,09 & 20,79 & 6,10 & ,613 & 0,540 \\
\hline \multirow{5}{*}{$\begin{array}{l}\text { ZKPQ-50- } \\
\text { CC }\end{array}$} & Ansiedad & 3,63 & 2,87 & 3,92 & 2,51 & 1,081 & 0,280 \\
\hline & Impulsividad & 7,75 & 2,65 & 6,75 & 2,12 & 4,332 & 0,000 \\
\hline & Actividad & 5,74 & 2,26 & 4,22 & 2,73 & 5,468 & 0,000 \\
\hline & Sociabilidad & 6,78 & 2,53 & 6,24 & 2,30 & 2,197 & 0,028 \\
\hline & Agresividad & 5,65 & 2,77 & 4,99 & 2,44 & 2,536 & 0,011 \\
\hline
\end{tabular}

Tabla 2

Correlaciones entre las escalas de afectos, humor y personalidad

\begin{tabular}{|c|c|c|c|c|c|c|c|}
\hline & & \multicolumn{4}{|c|}{ HSQ } & \multicolumn{2}{|c|}{ PANAS } \\
\hline & & Afiliación & Automejora & Agresividad & Autodestructivo & Negativo & Positivo \\
\hline \multirow{5}{*}{ ZKPQ 50-CC } & Neuroticismo/ansiedad & $-0,157 * *$ & $-0,234 * *$ & $-0,077^{*}$ & $0,232 * *$ & $0,547 * *$ & $-0,254 * *$ \\
\hline & Impulsividad & $0,329 * *$ & $0,220 * *$ & $0,361 * *$ & $0,270 * *$ & $0,105 * *$ & $0,090 * *$ \\
\hline & Actividad & 0,058 & $0,154 * *$ & 0,010 & 0,064 & 0,034 & $0,442 * *$ \\
\hline & Sociabilidad & $0,306 * *$ & $0,218 * *$ & $0,258 * *$ & $0,150 * *$ & $-0,111 * *$ & $0,110 * *$ \\
\hline & Agresividad/hostilidad & $0,253 * *$ & $0,122 * *$ & $0,368 * *$ & $0,171 * *$ & $0,179 * *$ & $-0,010$ \\
\hline \multirow{2}{*}{ PANAS } & Afectos negativos & $-0,159 * *$ & $-0,130 * *$ & 0,040 & $0,189 * *$ & & \\
\hline & Afectos positivos & $0,139 * *$ & $0,290 * *$ & 0,016 & $-0,011$ & & \\
\hline
\end{tabular}

$* \mathrm{p}<0,05 * * \mathrm{p}<0,01$ 
por 293 sujetos (44,93\%), con un menor índice de ansiedad y de afectos negativos, así como con un mayor uso de afectos positivos y mayor utilización del humor positivo (afiliativo y automejora) y puntuaciones altas en impulsividad, actividad y sociabilidad; (ii) con 171 sujetos $(26,22 \%$ ) que mostraban niveles más bajos de afectos (positivos y negativos), una menor actividad y agresividad, un menor empleo del humor de automejora y autodestructivo y niveles más altos de utilización del humor afiliativo; y (iii) con 188 participantes $(28,83 \%)$ en los que se encontraron niveles más altos de afectos negativos, mayor empleo del humor autodestructivo, ansiedad y agresividad, así como niveles más bajos de utilización de los afectos positivos, una menor impulsividad, actividad y sociabilidad y puntuaciones más bajas en el humor afiliativo y de automejora.

\section{Discusión y conclusiones}

En la investigación se encontraron un gran número de correlaciones entre la escala utilizada para medir sentido del humor (HSQ), la escala de afectos (PANAS) y la escala de personalidad (ZKPQ-50-CC), lo que indicaría la relación entre estas variables.

Los datos indican cómo los hombres hacen un uso más elevado de los distintos estilos del humor, tanto positivos como negativos, así como una mejor capacidad de reconocer y regular sus afectos, en línea con algunos estudios recientes (Dick \& Holtzman, 2013; Walther \& Hilbert, 2015). También se encontró que los hombres mostraban valores más altos en afecto negativo, siendo similares los resultados en cuanto a género y afecto positivo. Esto supone una diferencia con otros estudios que no encontraron diferencias en cuanto al uso los afectos en cuestión de género (Batool, Niazi \& Ghayas, 2014; Hay, 2000; Kotthoff, 2006), o que señalaban un mejor manejo de los afectos en el caso de las mujeres (Extremera, Fernández-Berrocal \& Salovey, 2006), lo cual indicaría que no existen suficientes estudios sobre este tema como para poder extraer conclusiones firmes acerca de la relación del género con los afectos y el uso del sentido del humor. En el apartado de los afectos,

Tabla 3

Centro de conglomerados finales de las variables sentido de afectos, humor y personalidad

\begin{tabular}{llrrrrrr}
\hline & & 1 & 2 & 3 & x & IC $95 \%$ & d.s. \\
\hline \multirow{2}{*}{ PANAS } & Afectos negativos & 18,93 & 16,28 & 28,07 & 20,86 & $20,37-21,36$ & 6,48 \\
& Afectos positivos & 37,76 & 27,32 & 28,69 & 32,40 & $31,91-32,88$ & 6,32 \\
\hline \multirow{2}{*}{ HSQ } & Afiliativo & 32,37 & 32,80 & 30,05 & 31,81 & $31,46-32,15$ & 4,45 \\
& Automejora & 28,46 & 24,64 & 24,74 & 26,38 & $25,99-26,77$ & 5,10 \\
& Agresivo & 18,86 & 19,33 & 18,88 & 18,98 & $18,68-19,28$ & 3,93 \\
& Autodestructivo & 16,81 & 16,33 & 17,89 & 16,99 & $16,64-17,34$ & 4,56 \\
\hline \multirow{3}{*}{ ZKPQ-50-CC } & Ansiedad & 2,9 & 3,2 & 6,1 & 3,78 & $3,67-4,07$ & 2,58 \\
& Ampulsividad & 7,1 & 6,8 & 6,7 & 6,92 & $6,74-7,09$ & 2,25 \\
& Actividad & 5,5 & 3,1 & 4,1 & 4,48 & $4,27-4,69$ & 2,71 \\
& Sociabilidad & 6,7 & 6,7 & 5,5 & 6,33 & $6,15-6,51$ & 2,35 \\
N. ${ }^{\circ}$ & Agresividad & 5,0 & 4,8 & 5,5 & 5,10 & $4,91-5,29$ & 2,51 \\
Pujetos & & 293 & 171 & 188 & & & \\
\hline
\end{tabular}

*Valores por debajo de la media

*Valores próximos a la media

*Valores por encima de la media 
se señalan dos cuestiones, por un lado, el bajo índice de sujetos con afectos extremadamente positivos; $y$, por otra parte, el índice tan alto tanto de hombres $(4,50 \%)$, como de mujeres $(7,20 \%)$ con afectos extremadamente negativos, tratándose de universitarios sin características ni historia psicopatológica previa.

En cuanto a la relación entre afectos, uso del humor y personalidad, se encontró correspondencia de los tipos de humor con todos los factores de la personalidad, con puntuaciones similares a estudios anteriores (Goma \& Valero, 2008). Caso especial es la ansiedad, cuyos resultados indican que, a mayores indicadores de esta, menor uso del humor. En los aspectos referentes a los afectos, su expresión correlaciona con los estilos de humor afiliativo, de automejora y autodestructivo, mientras que no se encontró relación con el humor agresivo. En el caso del afecto negativo, se comprobó que correlaciona negativamente con los humores positivos (afiliación y automejora), es decir, cuanto mayor expresión del afecto negativo tiene la persona, hay un menor uso de los tipos de humor positivos.

Estos resultados se pueden explicar, dado que el humor afiliativo (o humor social) mide el uso benigno del humor para facilitar las relaciones interpersonales (Erickson \& Feldstein, 2007), lo que implicaría su relación con la gestión de los afectos, en línea de los datos encontrados en otras investigaciones anteriores (Kuiper \& McHale, 2009). El humor de automejora, que mide el empleo de una visión humorística pero realista de la vida y que se usa como una estrategia de afrontamiento para regular los afectos negativos (Erickson \& Feldstein, 2007; Saroglou \& Scariot, 2002) mantiene correspondencia con el afecto positivo, señal del grado en el que una persona se siente activa, alerta, con energía y entusiasmo (Sandín, 1999), mientras que con el afecto negativo, que indica estados emocionales adversos (disgusto, ira, culpa, miedo y nerviosismo (Watson et al., 1988), se aprecia cómo a mayores índices de este afecto, menor uso de los humores positivos (afiliativo y de automejora).

El humor agresivo, que está relacionado directamente con hostilidad y agresividad (Kuiper \& McHale, 2009) no mostró correlaciones con los afectos, pero sí con todas las subescalas de personalidad a excepción de actividad (necesidad de actividad general).

Igualmente, el humor de descalificación personal, o autoagresivo, que busca el uso de un humor cínico a expensas de la propia persona mostró correlaciones con las subescalas de personalidad, a excepción de actividad, pero solo con el afecto negativo.

Por otra parte, en el análisis de clúster, se obtuvieron tres grupos: uno con un menor índice de ansiedad y afectos negativos, así como con un mayor uso de afectos positivos y de utilización del humor positivo (afiliativo y automejora), mayor impulsividad, actividad y sociabilidad; otro con niveles más bajos de afectos (positivos y negativos) un menor empleo del humor de automejora y autodestructivo y una menor actividad y agresividad, como también unos niveles más altos de utilización del humor afiliativo y agresivo y una más alta sociabilidad; y un tercero con niveles más altos de afectos negativos, empleo del humor autodestructivo, mayor ansiedad y agresividad, así como niveles más bajos de utilización de afectos positivos, humor afiliativo y de automejora y una menor impulsividad, actividad y sociabilidad. Estos resultados, indican que los afectos correlacionarían con los diferentes componentes tanto del humor como de la personalidad, siendo los afectos los que en mayor medida y en la relación adecuada se asocian con los distintos factores de estos constructos, cuestión apuntada por algunos estudios (Abel, 2002; Dionigi \& Gremigni, 2013; Edgar et al., 2012). Así, un buen uso de los afectos y del humor puede proporcionar soporte emocional y servir en la gestión de conflictos.

Los resultados deben ser interpretados teniendo en cuenta las limitaciones de este estudio, que, si 
bien la muestra es relevante a nivel estadístico, debería ampliarse a otros estratos de la población, incluidas poblaciones clínicas, donde podría darse una mayor asociación entre estos tres constructos: afectos, humor y personalidad. También serían deseables estudios longitudinales que permitiesen valorar la evolución de los afectos, el humor, así como su vinculación con la personalidad, a lo largo de un periodo más largo.

Por tanto, puede deducirse que los constructos de los afectos, sentido del humor y personalidad establecen adecuadas correlaciones, y al proporcionar los datos de la investigación, evidencia de la relación entre estas variables, se puede concluir que esto puede suponer la primera prueba de la existencia de una relación entre los afectos, el sentido del humor y la personalidad.

\section{Referencias}

Abel, M. H. (2002). Humor, stress and coping strategies. Humor, 15(4), 365-381.

Aillaud, M., \& Piolat, A. (2013). Compréhension et appreciation de l'humour: aproche cognitivo-émotionnelle. Psychologie française, 58, 255-275. doi: http://dx.doi.org/10.1016/j.psfr.2013.04.001

Aluja, A., Rossier, J., García L. F., Angleitner, A., Kuhlman, M., \& Zuckerman, M. (2006). A cross-cultural shortened form of the ZKPQ (ZKPQ-50-CC) adapted to English, French, German, and Spanish languages. Personality and Individual Differences, 41, 619-628. doi: http:// dx.doi.org/10.1016/j.paid.2006.03.001

Baquero, B. C., \& Jáuregui, E. (2006). Emociones positivas: humor positivo. Papeles del psicólogo, 27(1), 18-30.

Batool, M., Niazi, S., \& Ghayas, S. (2014). Emotional intelligence as a predictor of sense of humor and hope among adults. Journal of the Indian Academy of Applied Psychology, 40(2), 270-278.

Cann, A., \& Collett, C. (2014). Sense of humor, stable affect, and psychological well-being. Europe's
Journal of Psychology, 10(3), 464-479. doi: http://dx.doi.org/10.5964/ejop.v10i3.746

Capps, D. (2006). The psychological benefits of humor. Pastoral Psychology, 54(5), 393-411.

Cassaretto, M., \& Martínez, P. (2009). Validación de la Escala del Sentido del Humor en estudiantes universitarios. Revista de Psicología, 27(2), 287-309.

Clark, L. A., \& Watson, D. (1991). Tripartite Model of Anxiety and Depression: Psychometric evidence and taxonomic implications. Journal of Abnormal Psychology, 100(3), 316-336.

Crawford, J. R., \& Henry, J. D. (2004). The Positive and Negative Affect Schedule (PANAS): Construct validity, measurement properties and normative data in a large non-clinical sample. British Journal of Clinical Psychology, 43(1), 245-265. http://dx.doi.org/10.1348/0144665031752934

Dionigi, A., \& Gremigni, P. (2013). The psychology of humor. En Gremigni (Ed.) Humor and Health Promotion (pp. 1-14). Waltham, USA: Nova Medical.

Erickson, S., \& Feldstein, S. (2007). Adolescent humor and its relationship to coping, defense strategies, psychological distress, and well-being. Child Psychiatry and Human Development, 37, 255-271.

Fernández-Abascal, E. G. (2009). Emociones Positivas. Madrid: Editorial Pirámide.

Gignac, G. E., Karatamoglou, A., Wee, S., \& Palacios, G. (2014). Emotional intelligence as a unique predictor of individual differences in humour styles and humour appreciation. Personality and Individual Differences, 56, 34-39. doi: http:// dx.doi.org/10.1016/j.paid.2013.08.020

Goma, M., \& Valero, S. (2008). Spanish normative data of the Zuckerman-Kuhlman Personality Questionnaire in a general population sample. Psicothema, 20, 324-330. Recuperado de http:// hdl.handle.net/11162/4658

Hay, J. (2000). Emotions of humor in the conversations of men and women. Journal of Pragmatics, 
32, 709-742. doi: http://dx.doi.org/10.1016/j. paid.2014.02.024

Kotthoff, H. (2006). Gender and humor: the state of the art. Journal of Pragmatics, 38(1), 4-25. doi: http://dx.doi.org/10.1016/j.pragma.2005.06.003

Kozbetz, A., \& Nishioka, K. (2010). Humor comprehension, humor production, and insight: An exploratory study. Humor: International Journal of Humor Research, 23, 375-401. doi: http://dx.doi.org/10.1515/humr.2010.017

Kuiper, N. A. (2014). Investigating the role of humor in psychological health and well-being. Europe's Journal of Psychology, 10(3), 464-479. doi: http://dx.doi.org/10.5964/ejop.v10i3.809

Kuiper, N. A., \& McHale, N. (2009). Humor styles as mediators between self-evaluative standards and psychological well-being. Journal of Psychology, 143(4), 359-376. doi: http://dx.doi. org/10.3200/JRLP.143.4.359-376

Kuiper, N. A., Grimshaw, M., Leite, C., \& Kirsh, G. (2004). Humor is not always the best medicine: specific components of sense of humor and psychological well-being. Humor: International Journal of Humor Research, 17, 135-168. doi: http://dx.doi.org/10.1515/humr.2004.002

Longo, Y. (2015). The simple structure of positive affect. Social Indicators Research (en prensa). doi: http://dx.doi.org/10.1007/s11205-0140776-6

Martin, R. A. (2001). Humor, laughter, and physical health: methodological issues and research findings. Psychological Bulletin, 127(4), 504-519.

Martin, R. A., Puhlik-Doris, P., Larsen, G., Gray, J., \& Weir, K. (2003). Individual differences in uses of humor and their relation to psychological well-being: Development of the humor styles questionnaire. Journal of Research in Personality, 37, 48-75.

Panish, J. (2002). Life satisfaction in the elderly: the role of sexuality, sense of humor, and health. Dissertation Abstracts International, 63 (5-B).

Saroglou, V., \& Scariot, C. (2002). Humor Style Questionnaire: Personality and educational correlates in Belgian high school and college students. European Journal of Personality, 16, 43-54.

Walther, M., \& Hilbert, A. (2015). Emotional openness in overweight and normal-weight adolescents. Swiss Journal of Psychology, 71(1), 2936. doi: http://dx.doi.org/10.1024/1421-0185/ a000145

Watson, D., \& Tellegen, A. (1985). Toward a consensual structure of mood. Psychological Bulletin, 98(2), 219-235.

Watson, D., Clark, L. A., \& Tellegen, A. (1988). Development and validation of brief measures of positive and negative affect: the PANAS scales. Journal of Personality and Social Psychology, 54(6), 1063-1070.

Yip, J. A., \& Martin, R. A. (2006) Sense of humor, emotional intelligence, and social competence. Journal of Research in Personality, 40, 1202-1220. doi: http://dx.doi.org/10.1016/j. jrp.2005.08.005

Zuckerman, M. (2005). Psychobiology of personality. Cambridge: Cambridge University Press. doi: http://dx.doi.org/10.1017/CBO9780511813733

Zuckerman, M., Kuhlman, D. M., Teta, P., Joireman, J., \& Kraft, M. (1993). A comparison of three structural models of personality: The big three, the big five, and the alternative five. Journal of Personality and Social Psychology, 65, 757-768. doi: http://dx.doi.org/10.1037/00223514.65 .4 .757
Recibido: Enero 26, 2016 Aprobado: Abril 05, 2017 
